\title{
Prospects for Top Quark Studies with CMS for up to
} $1 \mathrm{fb}^{-1}$

\author{
Thomas Peiffer ${ }^{* \dagger}$ \\ Institut für Experimentelle Kernphysik, Karlsruhe Institute of Technology \\ Wolfgang-Gaede-Str. 1, 76131 Karlsruhe, Germany \\ E-mail: thomas.peifferakit.edu
}

The first long physics run of LHC is currently taking place at a center-of-mass energy of $7 \mathrm{TeV}$, and expected to go on until an integrated luminosity of 1 inverse femtobarn will have been collected. The CMS potential for top quark studies with this initial dataset is discussed, with emphasis on searches for new particles decaying into top-antitop quark pairs, measurements of the heavy flavour content of top-antitop quark events and the CKM matrix element $V_{\mathrm{tb}}$, as well as measurements of single top quark production.

The Xth Nicola Cabibbo International Conference on Heavy Quarks and Leptons, October 11-15, 2010

Frascati (Rome) Italy

\footnotetext{
*Speaker.

$\dagger$ on behalf of the CMS collaboration
} 


\section{Introduction}

The top quark is the heaviest known elementary particle. It was first discovered in 1995 in proton antiproton collisions by both Tevatron experiments CDF [1] and D0 [2]. The CMS experiment [3] at the Large Hadron Collider (LHC) at CERN has observed recently first candidate events which indicate top quark pair production in proton-proton collisions at a center-of-mass energy of $7 \mathrm{TeV}$ and performed a first cross section measurement [4]. At hadron colliders, the top quark is dominantly produced in pairs via the strong interaction. Single top quarks can also be produced in electroweak interactions. Due to its high mass it decays instantaneously into a b quark and a W boson before hadronization. Top quark pairs are classified from the decay of the W boson. Both $\mathrm{W}$ bosons can decay into hadrons, one can decay into leptons and the other one into hadrons, or both $\mathrm{W}$ bosons can decay into leptons. The instantaneous decay makes the top quark a challenging but also the only candidate to proof the properties of bare quarks. In 2011 the LHC will run with a center-of-mass energy of at least $7 \mathrm{TeV}$ and might accumulate data with an integrated luminosity of up to one inverse femtobarn. With such an amount of data, it will be possible to perform many measurements probing the properties of top quark production and decay in great detail.

Top quark properties are an interesting field to probe the Standard Model (SM) and to search for effects of beyond-SM effects. The measurement of single top quark production and also the determination of the heavy flavor content in top quark decays are suitable to extract the CKM matrix element $V_{\mathrm{tb}}$. In the production of top quark pairs unknown exchange particles might show up as resonances in the invariant mass spectrum of the top-antitop quark system.

\section{Search for single top quark production}

Single top quark production in electroweak interactions was first observed in 2009 at the Tevatron $[5,6]$. Measuring the top quark production cross section gives direct access to the CKM matrix element $V_{\mathrm{tb}}$, since the cross section is directly proportional to $\left|V_{\mathrm{tb}}\right|^{2}$. Three production channels are distinguished: The t-channel, the s-channel, and associated production together with a $\mathrm{W}$ boson. At the LHC the single top quark production is dominated by the t-channel.

The study of prospects of single top quark searches at CMS a Monte-Carlo based study [7] is performed assuming a scenario of a center-of-mass energy of $10 \mathrm{TeV}$ and integrated luminosities of the order of a few $100 \mathrm{pb}^{-1}$. An event selection is developed using the event signature of single top quark events with a muon and a jet from the top-quark decay. The main backgrounds to single top quark production are top quark pair production, $\mathrm{W} / \mathrm{Z}$ boson production in association with jets, and QCD multi-jet events. Events with exactly one isolated muon exceeding a transverse momentum $p_{\mathrm{T}}>20 \mathrm{GeV} / \mathrm{c}$ and two jets with $p_{\mathrm{T}}>30 \mathrm{GeV} / \mathrm{c}$ are selected. One of the two selected jets is required to have been identified as $a b$ jet using a track counting $b$ tagging algorithm. Furthermore, to the transverse mass of the reconstructed $\mathrm{W}$ boson has to be larger than $50 \mathrm{GeV} / \mathrm{c}^{2}$ to reduce QCD multi-jet backgrounds.

After the events selection, the expected signal over background ratio is still of the order of 1:2. For further discrimination, additional variables with different shapes for single top quark production compared to the backgrounds are needed. One candidate originates from the top quark polarization. Due to the V-A structure of the weak interaction, the top quark is to almost $100 \%$ left- 
handed. This results in angular correlations between the top quark decay products. For single top quark production the differential cross section is linear proportional $\cos \theta_{\mathrm{lj}}^{*}$, where $\theta_{\mathrm{lj}}^{*}$ is the angle between the direction of the outgoing lepton and the top spin axis, approximated by the direction of the untagged jet, in the top-quark rest frame. All backgrounds have a flat shape in the distribution of $\cos \theta_{\mathrm{lj}}^{*}$ and can therefore be distinguished from the single top quark signal, which is illustrated in figure 1 .

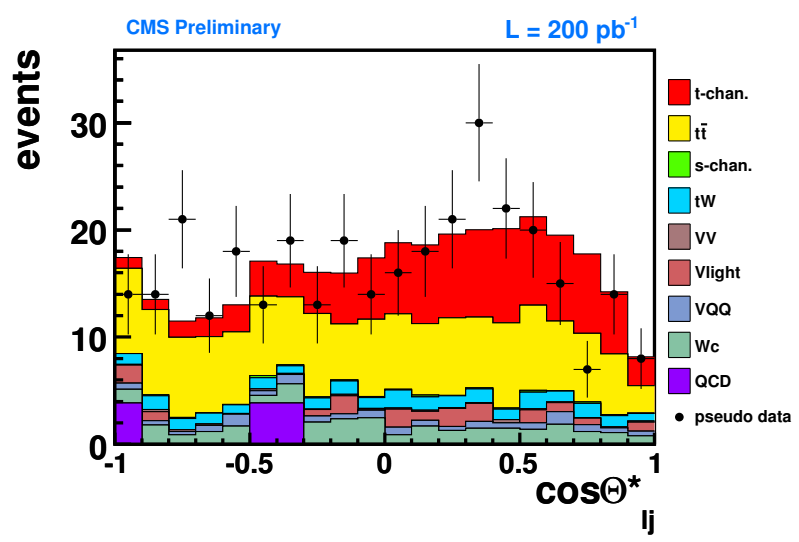

Figure 1: Cosine of the angle between charged muon and untagged jet, in the reconstructed top quark rest frame after full event selection.

The single top quark cross section is extracted from a template likelihood fit to the distribution of $\cos \theta_{\mathrm{lj}}^{*}$. In ensemble tests the expected statistical uncertainty and sensitivity are determined. With an integrated luminosity of $200 \mathrm{pb}^{-1}$ the expected statistical sensitivity is $2.8 \sigma$. The impact of systematic uncertainties on jet and missing transverse energy scales, b-tagging efficiency and mistagging rate, initial and final state radiation, and uncertainties due to the modelling of the parton distribution functions to the rate and shape of the fit templates are evaluated. It is found, that the usage of $\cos \theta_{\mathrm{lj}}^{*}$ as fit variable is quite robust against the influence of systematic uncertainties. The expected sensitivity is reduced to $2.7 \sigma$ accounting for these. A $5 \sigma$ observation is expected to be claimed at $700 \mathrm{pb}^{-1}$ at a center-of-mass energy of $10 \mathrm{TeV}$ fitting the distribution of $\cos \theta_{\mathrm{lj}}^{*}$ only. A combination of several variables which are sensitive to single top quark production will probably give the opportunity to observe single top quarks at CMS with a smaller dataset, even with a lower center-of-mass energy of $7 \mathrm{TeV}$.

\section{Measurement of the heavy flavor content}

The Standard Model predicts a decay of the top quark into $\mathrm{b}$ quark plus a W boson to nearly $100 \%$, since the CKM matrix element $V_{t b}$ is predicted to be close to 1 . In two studies $[8,9]$ the prospects for a measurement of the ratio $R=B(\mathrm{t} \rightarrow \mathrm{Wb}) / B(\mathrm{t} \rightarrow \mathrm{Wq})$ is performed using top quark pair candidates. Under the assumption of three quark generations, $R$ is equal to $\left|V_{t b}\right|^{2}$. The main challenge of these analyses is to identify jets originating directly from top quark decays and not from background events or hadronically decaying $\mathrm{W}$ bosons or additional radiation in top quark events. Knowing the $\mathrm{b}$ tagging efficiency for these jets from an independent measurement, it is possible to estimate $R$ from the number of $\mathrm{b}$ tags $n_{\mathrm{b}-\operatorname{tag}}$ observed in top quark pair events. 
The first analysis [8] selects events in the dilepton decay channel with e+ $\mu$. Both leptons must exceed $20 \mathrm{GeV} / \mathrm{c}$ in $p_{\mathrm{T}}$ and are required to have opposite charge. In addition, only events with two jets with $p_{\mathrm{T}}>30 \mathrm{GeV} / \mathrm{c}$ and missing transverse energy of at least $30 \mathrm{GeV}$ are selected. In the selected sample not all observed jets originate from top quark decays. About $10 \%$ of the remaining events are background events, but also jets in real top pair events might originate from initial or final state radiation. In the dilepton channel a charged lepton is expected to originate from a top quark decay. The fraction of misassigned jets can therefore be estimated from the distribution of the invariant mass of lepton-jet pair $M_{1, \mathrm{j}}$. For correctly assigned jets, $M_{1, \mathrm{j}}$ should not exceed $\sqrt{m_{t}^{2}-m_{W}^{2}}=156 \mathrm{GeV} / \mathrm{c}^{2}$. The fraction of misassigned jets not stemming from top quarks is estimated from the tail with $M_{1, j}>190 \mathrm{GeV} / \mathrm{c}^{2}$ (see figure $2 \mathrm{a}$ ). Two methods to construct the $M_{1, \mathrm{j}}$ distribution for misassignments from data are considered: swapping the jet in the lepton-jet pair with a jet from a different event, or randomly rotate the lepton momentum vector. Knowing the shape of the distribution in the tail region of $M_{1, j}$, allows to extrapolate into the signal region and to estimate the fraction of misassignments there.

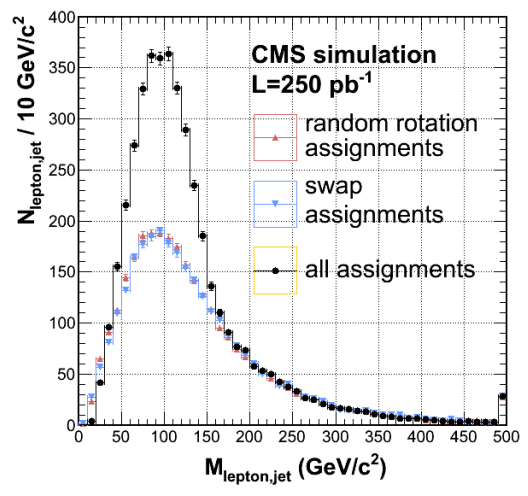

(a)

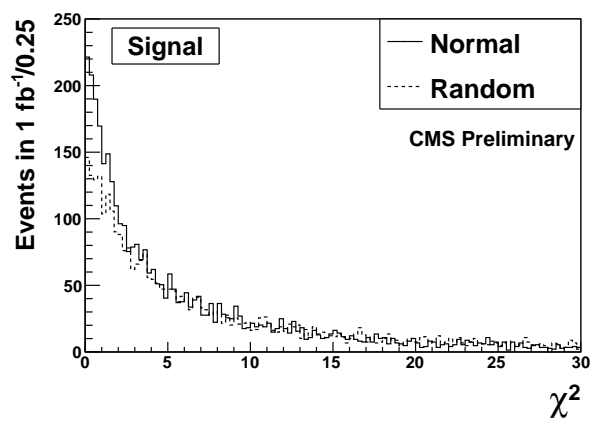

(b)

Figure 2: Discriminating variables to estimate the ratio of misassigned jets in the dilepton (a) and the lepton+jets analysis (b). The tails are dominated by wrong jet assignments, the shape of the distribution for misassignments are modeled by rotating or swapping jets randomly.

The second analysis [9] outlines the feasibility to measure $R$ in the lepton+jets decay channel. The correct assignment of jets to the direct decay products of the top quarks is here more challenging than in the dilepton decay mode. Events with one isolated muon or electron exceeding $p_{\mathrm{T}}>30$ $\mathrm{GeV} / \mathrm{c}$, at least four jets with $E_{\mathrm{T}}>40 \mathrm{GeV} / \mathrm{c}$, and centrality, which is the fraction of hard scattering going into the transverse plane, greater than 0.35 are selected. Furthermore, two jets are required to form an invariant mass close to the $\mathrm{W}$ mass. These jets are assigned to the hadronically decaying $\mathrm{W}$ boson. The remaining two jets have to be assigned to one of the top quarks. A $\chi^{2}$ variable including the reconstructed top quark masses is constructed for each assignment. The assignment of the two jets which minimises $\chi^{2}$ is chosen. The misassignment rate is estimated from the tail of the $\chi^{2}$ distribution with $\chi^{2}>4$ (see figure $2 b$ ). Wrong jet assignments are modeled by rotating the jet momenta randomly. The different shapes of the $\chi^{2}$ distributions for correct and wrong assignments makes it possible to subtract the fraction of misassignments.

In both anaylses $R$ is finally determined from the $n_{\mathrm{b}-\text { tag }}$ distribution. The main systematic uncertainty having to be taken into account is the uncertainty on the b tagging efficiency. In the 
dilepton channel, the expected overall uncertainty on $R$ is $9 \%$ assuming a scenario of $10 \mathrm{TeV}$ center-of-mass energy and an integrated luminosity of $250 \mathrm{pb}^{-1}$. In the lepton+jets analysis one can expect a statistical uncertainty of $12 \%$ and a systematic error of $11 \%$ on $R$ for the same centerof-mass energy but a higher luminosity of $1 \mathrm{fb}^{-1}$.

\section{Search for resonances in $M_{\mathrm{t} \bar{t}}$}

Many models beyond the Standard Model predict new heavy particles which preferable decay into top-antitop quark pairs. A narrow resonance will be visible in the invariant mass spectrum of the top quark pair $M_{\bar{t} \mathbf{t}}$. Three analyses $[10,11,12]$ were prepared to study the prospects for measurements of the $M_{\mathrm{t} \overline{\mathrm{t}}}$ spectrum.

Two analyses are performed in the muon plus jets decay channel. For the low mass region [10] with $M_{\mathrm{tt}} \lesssim 1 \mathrm{TeV} / \mathrm{c}^{2}$, an events selection requiring four jets with $p_{\mathrm{T}}>35 \mathrm{GeV} / \mathrm{c}$ and one muon with $p_{\mathrm{T}}>35 \mathrm{GeV} / \mathrm{c}$ is applied. The presence of four jets makes it possible to fully reconstruct the four momenta of all top quark decay products individually with a kinematic fitting algorithm. The top quark four momenta and $M_{\overline{\mathrm{t}}}$ are subsequently reconstructed by adding the momenta of the top quark decay products.

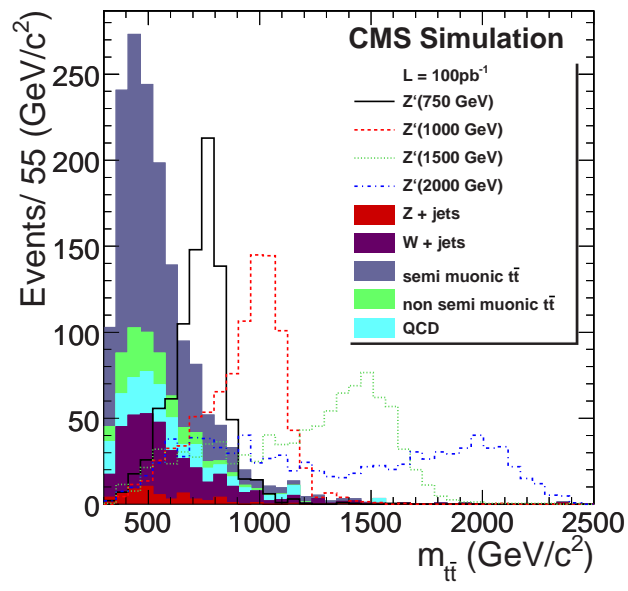

(a)

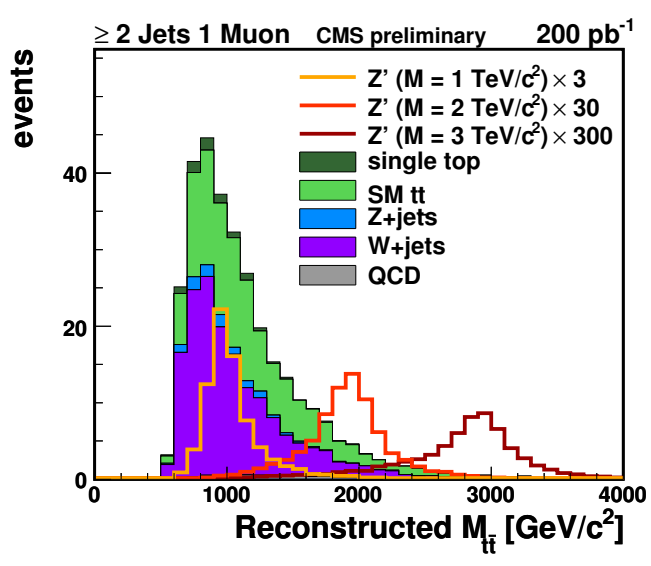

(b)

Figure 3: Reconstructed invariant mass distribution of the top quark pair in the low mass (a) and high mass muon+jets analysis (b) for backgrounds and hypothetic $Z$ ' resonances decaying into tt. $Z^{\prime} \rightarrow \mathrm{t}^{\mathrm{t}}$ is scaled to standard model $\overline{\mathrm{t}} \mathrm{t}$ cross section (a) and to multiples of a topcolor $Z^{\prime}$ [14] (b).

Searching for resonances in mass ranges $M_{\mathrm{tt}} \gtrsim 1 \mathrm{TeV} / \mathrm{c}^{2}$ requires different search strategies. If a high mass resonance decays into a pair of top quarks, the top quarks will have a large boost. Therefore, the decay products of the top quarks will almost merge together. Hadronically decaying top quarks will mostly end up in one merged jet in the detector, charged leptons from leptonically decaying top quarks will often be close to the corresponding b-jet from the top quark decay and will not be well isolated. Thus, the analysis searching for high mass resonances in the muon plus jets channel [11] selects events with at least two jets, a leading jet with $p_{\mathrm{T}}>200 \mathrm{GeV} / \mathrm{c}$ and further jets with $p_{\mathrm{T}}>50 \mathrm{GeV} / \mathrm{c}$. Instead of requiring isolation cuts for the muon, events with $\Delta R(\mu$, jet $)<0.4$ and $p_{\text {Trel }}(\mu$, jet $)<35 \mathrm{GeV} / \mathrm{c}$ are vetoed. In addition, a cut on the scalar sum of $p_{\mathrm{T}, \mu}$ and missing 
transverse energy at $200 \mathrm{GeV}$ is applied. The reconstruction of the top quark four momenta is done by assigning jets either to the hadronically or leptonically decaying top quark. The expected event topology of two top quarks in back-to-back configuration with all decay products close together is utilized to find an optimal jet assignment. The reconstructed $M_{\mathrm{t}}$ distributions from both muon+jets studies for backgrounds and possible signals are shown in figure 3 for luminosities of 100 to 200 $\mathrm{pb}^{-1}$ and $10 \mathrm{TeV}$ center-of-mass energy.

A third analysis [12] searches for boosted top quarks in the full hadronic decay channel. The expected event topology consists of two hard jets. To distinguish boosted top quark events from QCD di-jet production, a so called top tagging algorithm is applied. The top tagging algorithm searches for specific substructures in jets. A jet from the hadronic decay of top quarks should consist of three quark sub-jets, where two sub-jets form an invariant mass compatible with a W boson mass and all three added sub-jets have an invariant mass close to the top quark mass. In a first step, a Cambridge-Aachen jet algorithm [13] with a cone radius of 0.8 is applied to find two jets with $p_{\mathrm{T}}>250 \mathrm{GeV} / \mathrm{c}$. Since the Cambridge-Aachen algorithm is a sequential clustering algorithm, the last clustering steps can be reversed to find sub-jets. Finally, only events where both jets can be decomposed into sub-jets with specific invariant masses are selected.

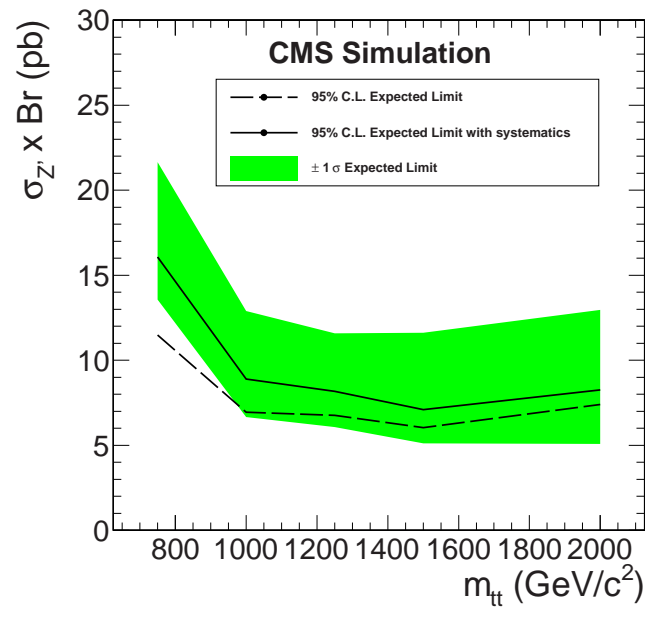

(a)

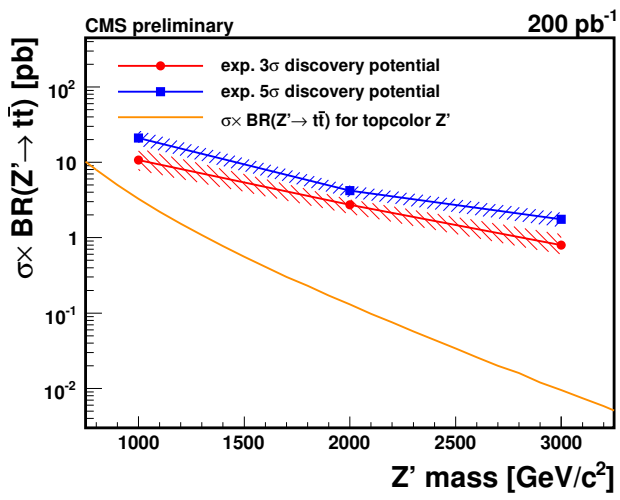

(b)

Figure 4: Expected $2 \sigma$ exclusion limits in the low-mass muon+jets channel (a), the high mass muon+jets channel (b). Reference model is a topcolor Z' [14].

For the muon+jets analyses, the expected exclusion limits for narrow resonances decaying into top quark pairs are determined in template likelihood fits to the reconstructed invariant mass $M_{\overline{\mathrm{t}}}$. Constraints to the backgrounds are taken from appropriate sidebands. The systematic uncertainties on jet energy scale, parton distribution function, initial and final state radiation, matching threshold between hard matrix element generation and parton showering, and factorization scale are incorporated into the fit. The main systematic uncertainty is found to be the uncertainty on the jet energy scale. In the top tagging analysis the expected limits on resonance cross sections are calculated by counting events in specific mass windows. The dominant systematic uncertainty for this measurement is related to the top-quark tagging efficiency. The results of all three analyses are shown in figures 4 and 5. The expected limits on resonances in top quark pair production, which might be given in 2011, are of the order of a few pb up to masses of several $\mathrm{TeV} / \mathrm{c}^{2}$. 


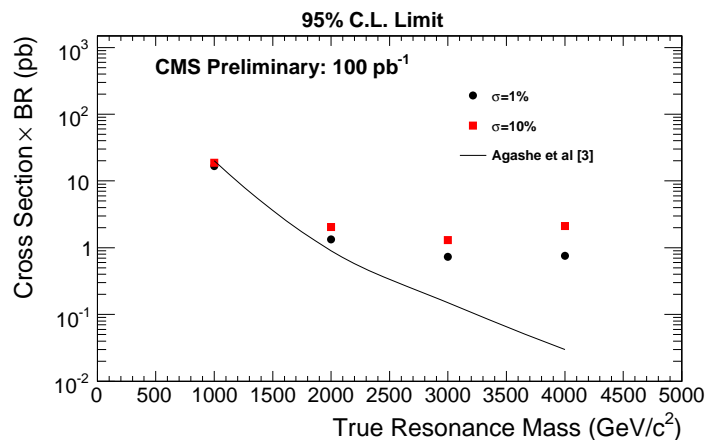

Figure 5: Expected $2 \sigma$ exclusion limits in the full hadronic analysis. Reference model is a Kaluza Klein gluon in a Randall Sundrum model [15].

\section{References}

[1] F. Abe et al. [CDF Collaboration], Observation of top quark production in $\bar{p} p$ collisions, Phys. Rev. Lett. 74 (1995) 2626 [arXiv:hep-ex/9503002].

[2] S. Abachi et al. [D0 Collaboration], Observation of the top quark, Phys. Rev. Lett. 74 (1995) 2632 [arXiv:hep-ex/9503003].

[3] CMS Collaboration, The CMS experiment at the CERN LHC, JINST 3 (2008) S08004.

[4] CMS Collaboration, First Measurement of the Cross Section for Top-Quark Pair Production in Proton-Proton Collisions at $\sqrt{s}=7 \mathrm{TeV}$, arXiv:1010.5994 [hep-ex].

[5] V. M. Abazov et al. [D0 Collaboration], Observation of Single Top-Quark Production, Phys. Rev. Lett. 103 (2009) 092001 [arXiv:0903.0850 [hep-ex]].

[6] T. Aaltonen et al. [CDF Collaboration], First Observation of Electroweak Single Top Quark Production, Phys. Rev. Lett. 103 (2009) 092002 [arXiv:0903.0885 [hep-ex]].

[7] CMS Collaboration, Prospects for the measurement of the single-top $t$-channel cross section in the muon channel with $200 \mathrm{pb}^{-1}$ of CMS data at $10 \mathrm{TeV}$, CMS PAS TOP-09-005.

[8] CMS Collaboration, Probing the heavy flavor content of the t $\bar{t}$ dilepton channel in proton-proton collisions at $\sqrt{s}=10 \mathrm{TeV}$, CMS PAS TOP-09-001.

[9] CMS Collaboration, Plan for a $B(t \rightarrow W b) / B(t \rightarrow W q)$ measurement in $t \bar{t}$ semi-leptonic decays at $\sqrt{s}=10 \mathrm{TeV}$, CMS PAS TOP-09-007.

[10] CMS Collaboration, Search for narrow resonances in top-pair production close to threshold in the semileptonic muon channel at $\sqrt{s}=10 \mathrm{TeV}$, CMS PAS TOP-09-009.

[11] CMS Collaboration, Search for heavy narrow t⿳亠丷厂 resonances in muon-plus-jets final states with the CMS detector, CMS PAS EXO-09-008.

[12] CMS Collaboration, Search for High Mass ț̄ Resonances in the All-Hadronic Mode, CMS PAS EXO-09-002.

[13] Y. L. Dokshitzer, G. D. Leder, S. Moretti and B. R. Webber, Better Jet Clustering Algorithms, JHEP 9708 (1997) 001 [arXiv:hep-ph/9707323].

[14] R. M. Harris, C. T. Hill and S. J. Parke, Cross section for topcolor $Z_{t}{ }_{t}$ decaying to t anti-t, arXiv:hep-ph/9911288.

[15] K. Agashe, S. Gopalakrishna, T. Han, G. Y. Huang and A. Soni, LHC Signals for Warped Electroweak Charged Gauge Bosons, Phys. Rev. D 80 (2009) 075007 [arXiv:0810.1497 [hep-ph]]. 